\title{
A novel melanoma therapy stirs up a storm: ipilimumab-induced thyrotoxicosis
}

\author{
Christine $\mathrm{Yu}^{1}$, Inder J Chopra ${ }^{2}$ and Edward $\mathrm{Ha}^{1}$ \\ ${ }^{1}$ Department of Medicine ${ }^{2}$ Division of Endocrinology, Department of Medicine, University of California, \\ 757 Westwood Plaza Blvd, Suite 7501, Los Angeles, CA 90095, USA
}

Correspondence

should be addressed

to $\mathrm{C} Y u$

Email

Christineyu@

mednet.ucla.edu

\section{Summary}

Ipilimumab, a novel therapy for metastatic melanoma, inhibits cytotoxic T-lymphocyte apoptosis, causing both antitumor activity and significant autoimmunity, including autoimmune thyroiditis. Steroids are frequently used in treatment of immune-related adverse events; however, a concern regarding the property of steroids to reduce therapeutic antitumor response exists. This study describes the first reported case of ipilimumab-associated thyroid storm and implicates iopanoic acid as an alternative therapy for immune-mediated adverse effects. An 88-year-old woman with metastatic melanoma presented with fatigue, anorexia, decreased functional status, and intermittent diarrhea for several months, shortly after initiation of ipilimumab - a recombinant human monoclonal antibody to the cytotoxic T-lymphocyte-associated antigen 4 (CTLA4). On arrival, she was febrile, tachycardic, and hypertensive with a wide pulse pressure, yet non-toxic appearing. She had diffuse, non-tender thyromegaly. An electrocardiogram (EKG) revealed supraventricular tachycardia. Blood, urine, and stool cultures were collected, and empiric antibiotics were started. A computed tomography (CT) angiogram of the chest was negative for pulmonary embolism or pneumonia, but confirmed a diffusely enlarged thyroid gland, which prompted thyroid function testing. TSH was decreased at $0.16 \mu \mathrm{IU} / \mathrm{ml}$ (normal 0.3-4.7); free tri-iodothyronine $\left(T_{3}\right)$ was markedly elevated at $1031 \mathrm{pg} / \mathrm{dl}$ (normal 249-405), as was free thyroxine $\left(\mathrm{T}_{4}\right)$ at $5.6 \mathrm{ng} / \mathrm{dl}$ (normal 0.8-1.6). With iopanoic acid and methimazole therapy, she markedly improved within $48 \mathrm{~h}$, which could be attributed to lowering of serum $\mathrm{T}_{3}$ with iopanoic acid rather than to any effect of the methimazole. Ipilimumab is a cause of overt thyrotoxicosis and its immune-mediated adverse effects can be treated with iopanoic acid, a potent inhibitor of $\mathrm{T}_{4}$-to- $\mathrm{T}_{3}$ conversion.

\section{Learning points:}

- While ipilimumab more commonly causes autoimmune thyroiditis, it can also cause thyroid storm and clinicians should include thyroid storm in their differential diagnosis for patients who present with systemic inflammatory response syndrome.

- Immune-related adverse reactions usually occur after 1-3 months of ipilimumab and baseline thyroid function testing should be completed before initiation with ipilimumab.

- Conflicting data exist on the use of prednisone for treatment of CTLA4 adverse effects and its attenuation of ipilimumab's antitumor effect. Iopanoic acid may be considered as an alternative therapy in this setting.

\section{Background}

Developments in cancer therapeutics have shifted toward novel target-specific immunotherapies that act along signaling pathways. Ipilimumab, an antineoplastic agent used in unresectable metastatic melanoma, is a recombinant human $\mathrm{MAB}$ that acts against surface receptor cytotoxic T-lymphocyte-associated antigen 4 (CTLA4) to modulate immune response (1). Through 
its inhibition of CTLA4, ipilimumab disinhibits the proliferation of effector T-lymphocytes, leading to antitumor activity. It has been shown to prolong the overall survival in patients with metastatic melanoma, although the greatest impact appears to be in those with previously treated metastatic disease $(2,3)$. However, due to its enhancement of immunity, ipilimumab, similar to other agents including interferon $(4,5)$, has the potential for significant immune-mediated toxicity, including inflammatory colitis, dermatitis, and endocrinopathies $(2,6,7)$. Ipilimumab has been associated with autoimmune hypophysitis with an incidence of 5$17 \%$ (4) and a wide phenotypic spectrum of thyroid function abnormalities, most commonly, thyroiditis and/or hypothyroidism, in roughly $1-2 \%$ of patients (2), and less commonly orbitopathy and subclinical hyperthyroidism $(2,3,4,5,8,9)$. We describe herein a case of ipilimumab-induced thyroid storm in a patient with metastatic melanoma. To our knowledge, severe hyperthyroidism has not yet been reported in the setting of treatment with ipilimumab.

\section{Case presentation}

An 88-year-old Caucasian female with a history of desmoplastic melanoma presented to the hospital in March 2012 with 3 months of fatigue, poor appetite, and failure to thrive. She had previously been treated with wide excision in November 2009, but was subsequently found to have diffusely metastatic melanoma of the liver, lungs, and bone on CT imaging in October 2011. She received rounds of ipilimumab on January 30 and

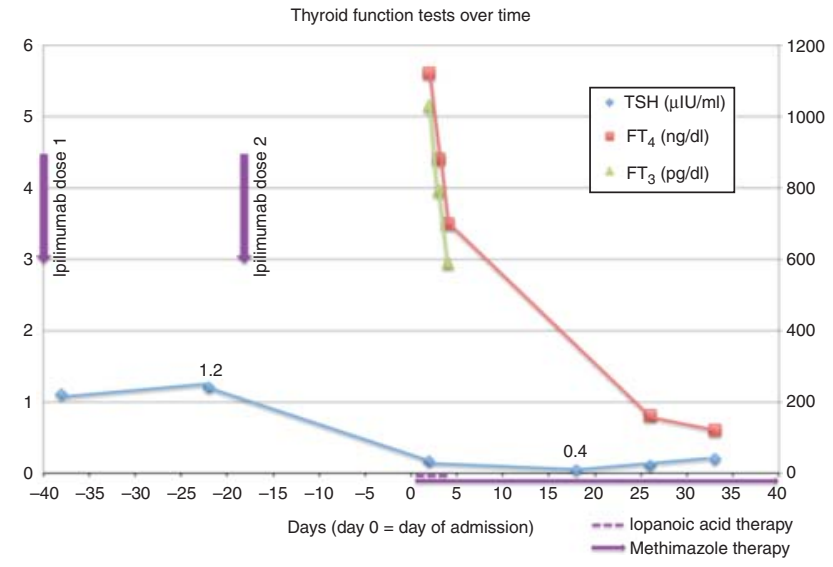

Figure 1

Thyroid function tests throughout the patient's course indicating a dramatic decline in free $T_{3}$ and free $T_{4}$ immediately after therapy with iopanoic acid and methimazole. Please note day 0 represents the day of admission.
February 21, 2012, with the second dose 3 weeks before admission. After starting immunotherapy, the patient developed progressive weakness, anorexia, and fatigue, and was unable to complete her activities of daily living. She also endorsed a 5-lb weight loss, anxiety, abdominal pain, and non-bloody diarrhea.

On presentation to the Emergency Department, her initial vital signs were significant for a temperature of $38.1^{\circ} \mathrm{C}$, heart rate of 105 b.p.m., and blood pressure of 144/64 mmHg. Two hours later, she developed a fever to $38.6{ }^{\circ} \mathrm{C}$, worsened hypertension with a wide pulse pressure to $173 / 75 \mathrm{mmHg}$, and tachycardia to the 150 b.p.m. Physical examination was notable for an elderly female with moist skin, tachycardia, and non-tender thyromegaly.

\section{Investigation}

Initial laboratory studies were significant for a white blood cell count of $6.6 \times 10^{3} / \mu \mathrm{l}$ with a normal differential and an elevated ionized calcium level at $1.35 \mathrm{mmol} / 1$ (1.09-1.29). An EKG demonstrated sinus tachycardia with frequent premature atrial contractions. Blood, urine, and stool cultures were collected, and empiric antibiotics with i.v. fluids were started. She was admitted to the general medicine service for the systemic inflammatory response syndrome with suspected occult infection in the setting of recent chemotherapy.

The first night of hospitalization, she developed intermittent chest pain and was found to have paroxysmal supraventricular tachycardia with a heart rate elevated to the 160 b.p.m. Her heart rhythm then converted to atrial fibrillation with rapid ventricular rate, requiring i.v. metoprolol and digitalis loading. Serial cardiac enzymes were negative. In the setting of her active malignancy, recent immobility, and unexplained tachycardia (modified Wells score $>4$ ), a CT angiogram of the chest was obtained and demonstrated metastatic melanoma, hydrostatic pulmonary edema, and diffuse thyroid gland enlargement. This latter finding prompted subsequent thyroid function testings. Her thyroid-stimulating hormone (TSH) in February 2012, before her second round of ipilimumab, was normal at $1.2 \mu \mathrm{IU} / \mathrm{ml}$. However, TSH on admission was low at $0.16 \mu \mathrm{IU} / \mathrm{ml}(0.3-4.7)$, free tri-iodothyronine $\left(\mathrm{FT}_{3}\right)$ elevated at $1031 \mathrm{pg} / \mathrm{dl}(249-405)$, and free thyroxine $\left(\mathrm{FT}_{4}\right)$ elevated at $5.6 \mathrm{ng} / \mathrm{dl}(0.8-1.6)$ (Fig. 1). Pan-cultures returned negative and an $0800 \mathrm{~h}$ cortisol was normal at $30 \mu \mathrm{g} / \mathrm{dl}$ (8-25). Ipilimumabinduced thyroiditis was suspected. Given the recent iodine contrast load from the CT angiogram, radioactive iodine 
uptake scan was not performed. A number of serologies including TPO antibody, thyroglobulin antibody, thyroidstimulating Ig, TSH-binding inhibitor, and TSH receptor antibody returned negative. Ultrasound of her thyroid demonstrated numerous small $(<3 \mathrm{~mm})$ cysts, but none amenable for fine-needle aspiration; there were no solid thyroid nodules.

\section{Treatment}

Given her overt thyrotoxicosis, she was started on iopanoic acid $500 \mathrm{mg}$ three times daily for 3 days and methimazole $10 \mathrm{mg}$ daily. Steroids were considered, but after consulting with the patient's oncologist, this option was deferred given the patient's acute illness and the possible abrogation of ipilimumab-induced antitumor activity. After $48 \mathrm{~h}$ of treatment with iopanoic acid and methimazole, her $\mathrm{FT}_{3}$ and $\mathrm{FT}_{4}$ had dropped to $590 \mathrm{pg} / \mathrm{dl}$ and $3.5 \mathrm{ng} / \mathrm{dl}$ respectively. She defervesced, had no further tachyarrhythmias, and her hypercalcemia resolved.

\section{Outcome and follow-up}

Three weeks after discharge, her TSH was still low at $0.11 \mu \mathrm{IU} / \mathrm{ml}$ but with a normal $\mathrm{FT}_{4}$ level of $0.8 \mathrm{ng} / \mathrm{dl}$. She was clinically euthyroid, and her methimazole was discontinued. Her digoxin and metoprolol were weaned off given improvement of her symptoms after thyroidsuppressive therapy. She was eventually placed on hospice care after staging scans demonstrated progression of the melanoma despite ipilimumab.

\section{Discussion}

This case, to our knowledge, highlights the first report of ipilimumab-induced thyroid storm. While improvement of the patient's thyroid function tests may have been the natural history of the adverse reaction, the brisk decline in $\mathrm{FT}_{3}$ when compared with $\mathrm{FT}_{4}$ is consistent with rapid inhibition of $\mathrm{T}_{4}$ to- $\mathrm{T}_{3}$ conversion observed with iopanoic acid therapy. In comparison, resolution of hyperthyroidism of Graves' disease with antithyroid medications such as methimazole would have taken much longer (weeks to months) before a substantial (clinical or laboratory) improvement.

Interestingly, the patient's initial presentation masqueraded as possible sepsis in the setting of recent immunotherapy, with two of the four systemic inflammatory reaction syndrome criteria including temperature $>38.3^{\circ} \mathrm{C}$ and heart rate $>90$ b.p.m., but not respiratory rate $>20$ breaths/min or white blood cell count $>12 \times 10^{3} / \mu$ l.
The parallel presentation raises the question of diagnostic criteria for thyroid storm that may help to differentiate sepsis from frank thyrotoxicosis. While Burch \& Wartofsky (10) first proposed a scoring system using clinical criteria including thermoregulatory, cardiovascular, and CNS dysfunction, currently no standardized, universally accepted, or validated clinical tools for diagnosis exist. According to this system, scores 45 or more, highly suggestive of thyroid storm, could also easily describe sepsis, as in our patient who had obtained a score of 70 (points for temperature of $38.6^{\circ} \mathrm{C}$, diarrhea, pulse $>140$ b.p.m., atrial fibrillation, and precipitant history). Hence, there is a need for a more specific scoring system. More recently, Akamizu et al. (11) have developed diagnostic criteria based on retrospective data from the largest epidemiological and outcomes data to date, in which thyroid storm was defined as thyrotoxicosis with a combination of fever, tachycardia, congestive heart failure, and/or gastrointestinal/hepatic manifestations. Thyroid storm, based on these criteria, has an annual incidence of 0.2 patients/ 100000 population and a mortality rate of $9.5-11 \%$. Limitations include the retrospective nature of the study and the strictly Japanese study population.

Akamizu et al. (11) identified irregular use or cessation of antithyroid drug therapy and infections as the most common triggers for thyroid storm. Our patient, however, had neither of these; rather, we believe she was exposed to two other possible triggers, the most likely of which is ipilimumab. The second trigger or exacerbating factor may have been the large iodine load from the CT angiogram, inducing a Jod Basedow effect in the setting of ipilimumab-induced autoimmunity. This phenomenon, also known as iodine-induced hyperthyroidism, occurs when excess iodine leads to a sustained increase in hormone synthesis and secretion by autonomous thyroid nodule, causing elevated thyroid levels (12). However, our patient showed no solid thyroid nodules, rendering the Jod Basedow phenomenon rather unlikely.

Given that our patient was acutely ill, iodine contrast agents and thionamides were selected for therapy. The use of prednisone was considered for treatment of CTLA4 adverse effects, but deferred out of the oncology team's concern that steroids may attenuate the antitumor effect of ipilimumab. There are conflicting data on the validity of this interaction $(13,14,15)$. Across three phase II studies for ipilimumab, 26 out of 117 patients achieved complete or partial response, or stable disease without disease progression (13). Of these 26 people, 11 of 14 people maintained a response after steroids when compared with 
nine of 12 who did not receive any steroids. Hence, the authors suggested that systemic steroids did not affect the development or maintenance of ipilimumab activity. Conversely, Weber et al. (16) found different results when they conducted a randomized, double-blinded, placebo-controlled phase II study on 115 patients comparing ipilimumab with and without prophylactic budesonide. The authors found that those treated with prophylactic budesonide had a decreased response rate of $12.1 \%$ and the median overall survival of 17.7 months compared with $15.8 \%$ and 19.3 months in the placebo group. While there is no definitive conclusion regarding the potential dampening effects of steroids on immunemediated antitumor activity, this remained a concern and we chose an alternative therapy for hyperthyroidism in our patient.

Based on a compilation of case report data, immunerelated adverse reactions usually occur after 1-3 months (2) or two to four sessions (16) of ipilimumab. Adverse events from treatment-related toxic effects may be confused with thyroid dysfunction, causing delay in dose adjustments or even cessation of therapy. Hence, while there are no official screening guidelines for thyroid function in patients undergoing ipilimumab therapy (4), thyroid function tests should be obtained before initiating treatment and monitored frequently, every 3 months, or sooner if the patient develops symptoms. Presently, treatment guidelines for immune-related adverse reactions per ipilimumab package insert and trial protocol revolve primarily around cessation of ipilimumab and the initiation of prednisone, dosed at $1-2 \mathrm{mg} / \mathrm{kg}$ per day. While there is little evidence to support the efficacy of this therapeutic regimen, the hypothesis that heightened autoimmunity leads to the endocrinopathies makes the aforementioned treatments logical choices. Data are limited in describing the successful treatment of asymptomatic hypo- and hyperthyroidism with steroids $(16,17,18)$. Our patient's prompt and successful response highlights the usefulness of iopanoic acid as an alternative therapy for hyperthyroidism. This treatment is not known to specifically target the immune system. Additionally, it is still unclear whether steroids abrogate ipilimumab-induced immune antitumor activity. Data comparing steroids and alternative treatment are needed to clarify management of ipilimumab-induced hyperthyroidism, including thyroid storm. Additionally, more prospective data are necessary to evaluate whether ipilimumab can be continued safely in patients who develop severe hyperthyroidism that responds to antithyroid medications.
Declaration of interest

The authors declare that there is no conflict of interest that could be perceived as prejudicing the impartiality of the research reported.

\section{Funding}

This research did not receive any specific grant from any funding agency in the public, commercial or not-for-profit sector.

\section{Patient consent}

The patient is deceased.

\section{Author contribution statement}

C Yu, MD contributed to clinical care of the patient and wrote the manuscript. I J Chopra, MD edited the manuscript and was a content expert. E Ha, MD contributed to clinical care of the patient and edited the manuscript.

\section{References}

1 Dillard T, Yedinak CG, Alumkal J \& Fleseriu M 2010 Anti-CTLA-4 antibody therapy associated autoimmune hypophysitis: serious immune related adverse events across a spectrum of cancer subtypes. Pituitary 13 29-38. (doi:10.1007/s11102-009-0193-z)

2 Hodi FS, O'Day SJ, McDermott DF, Weber RW, Sosman JA, Haanen JB, Gonzalez R, Robert C, Schadendorf D, Hassel JC et al. 2010 Improved survival with ipilimumab in patients with metastatic melanoma. New England Journal of Medicine 363 711-723. (doi:10.1056/NEJMoa 1003466)

3 Robert C, Thomas L, Bondarenko I, O'Day S, Weber J, Garbe C, Lebbe C, Baurain J-F, Testori A, Grob J-J et al. 2011 Ipilimumab plus dacarbazine for previously untreated metastatic melanoma. New England Journal of Medicine 364 2517-2526. (doi:10.1056/NEJMoa1104621)

4 Hamnvik OP, Larsen PR \& Marqusee E 2011 Thyroid dysfunction from antineoplastic agents. Journal of the National Cancer Institute 103 1572-1587. (doi:10.1093/jnci/djr373)

5 Jacobs EL, Clare-Salzler MJ, Chopra IJ \& Figlin RA 1991 Thyroid function abnormalities associated with the chronic outpatient administration of recombinant interleukin- 2 and recombinant interferon- $\alpha$. Journal of Immunotherapy 10 448-455. (doi:10.1097/00002371199112000-00009)

6 Wolchok JD, Neyns B, Linette G, Negrier S, Lutzky J, Thomas L, Waterfield W, Schadendorf D, Smylie M, Guthrie T, Jr et al. 2010 Ipilimumab monotherapy in patients with pretreated advanced melanoma: a randomized, double-blind, multicentre, phase 2, dose-ranging study. Lancet. Oncology 11 155-164. (doi:10.1016/ S1470-2045(09)70334-1)

7 Beck KE, Blansfield JA, Tran KQ, Feldman AL, Hughes MS, Royal RE, Kammula US, Topalian SL, Sherry RM, Kleiner D et al. 2006 Enterocolitis in patients with cancer after antibody blockade of cytotoxic T-lymphocyte-associated antigen 4. Journal of Clinical Oncology 24 2283-2289. (doi:10.1200/JCO.2005.04.5716)

8 Min L, Vaidya A \& Becker C 2011 Thyroid autoimmunity and ophthalmopathy related to melanoma biological therapy. European Journal of Endocrinology 164 303-307. (doi:10.1530/EJE-10-0833)

9 Bronstein Y, Ng CS, Hwu P \& Hwu WJ 2011 Radiologic manifestations of immune-related adverse events in patients with metastatic melanoma undergoing anti-CTLA-4 antibody therapy. AJR. American Journal of Roentgenology 197 W992-W1000. (doi:10.2214/AJR.10.6198) 
10 Burch HB \& Wartofsky L 1993 Life-threatening thyrotoxicosis. Thyroid storm. Endocrinology and Metabolism Clinics of North America 22 263-277.

11 Akamizu T, Satoh T, Isozaki O, Suzuki A, Wakino S, Iburi T, Tsuboi K, Monden T, Kouki T, Otani $\mathrm{H}$ et al. 2012 Diagnostic criteria and clinico-epidemiological features of thyroid storm based on a nationwide survey. Thyroid 22 661-679. (doi:10.1089/thy.2011.0334)

12 Wartofsky L 2012 Clinical criteria for the diagnosis of thyroid storm. Thyroid 22 659-660. (doi:10.1089/thy.2012.2207.ed1)

13 Amin A, DePril V, Hamid O, Wolchock J, Maio M, Neyns B, Chin K, Ibrahim R, Hoos A \& O'Day S 2009 Evaluation of the effect of systemic corticosteroids for the treatment of immune-related adverse events (irAEs) on the development or maintenance of ipilimumab clinical activity. Journal of Clinical Oncology 27 15s (suppl; abstr 9037). (doi:10.1200/JCO.2008.20.6235)

14 El-Shirbiny AM, Stavrou SS, Dnistrian A, Sonenberg M, Larson SM \& Divgi CR 1997 Jod-Basedow syndrome following oral iodine and radioiodinated-antibody administration. Journal of Nuclear Medicine $\mathbf{3 8}$ 1816-1817.
15 Harmankaya K, Erasim C, Koelblinger C, Ibrahim R, Hoos A, Pehamberger H \& Binder M 2011 Continuous systemic corticosteroids do not affect the ongoing regression of metastatic melanoma for more than two years following ipilimumab therapy. Medical Oncology 28 1140-1144. (doi:10.1007/s12032-010-9606-0)

16 Weber J, Thompson JA, Hamid O, Minor D, Amin A, Ron I, Ridolfi R, Assi H, Maraveyas A, Berman D et al. 2009 A randomized, double-blind, placebocontrolled, phase II study comparing the tolerability and efficacy of ipilimumab administered with or without prophylactic budesonide in patients with unresectable stage III or IV melanoma. Clinical Cancer Research 15 5591-5598. (doi:10.1158/1078-0432.CCR-09-1024)

17 Borodic G, Hinkle DM \& Cia Y 2011 Drug-induced graves disease from CTLA-4 receptor suppression. Ophthalmic Plastic and Reconstructive Surgery 27 e87-e88. (doi:10.1097/IOP.0b013e3181ef72a1)

18 Yang JC, Hughes M, Kammula U, Royal R, Sherry RM, Topalian SL, Suri KB, Levy C, Allen T, Mavroukakis S et al. 2007 Ipilimumab (anti-CTLA4 antibody) causes regression of metastatic renal cell cancer associated with enteritis and hypophysitis. Journal of Immunotherapy 30 825-830. (doi:10.1097/CJI.0b013e318156e47e)

Received in final form 14 January 2015

Accepted 26 January 2015 